\title{
MAGNETIC RESONANCE SIGNAL LOSS IN TURBULENT SHEAR FLOW
}

\author{
LIANG-DER JOU \\ Department of Radiology, Veteran Administration Medical Center \\ University of California at San Francisco, San Francisco, California, U.S.A.
}

\begin{abstract}
NMR signal loss due to turbulent shear flow is discussed, and a general expression for the phase fluctuation is derived. In the presence of flow shear, the velocity fluctuation perpendicular to the direction of magnetic gradient and the Reynolds stress can cause loss of MR signal. Most of signal loss results from the boundary layer. where the flow shear is strong in turbulent pipe flow. Half the signal loss within the mixing layer distal to a moderate stenosis is caused by the velocity fluctuation in the direction of magnetic gradient, while the remaining results from the velocity fluctuation perpendicular to the magnetic gradient. The use of eddy diffusivity for the description of signal dephasing in a spin echo sequence is also addressed: A positive, constant eddy diffusivity can not describe the temporal change of phase fluctuation correctly.
\end{abstract}

Biomed Eng Appl Basis Comm, 2002 (February); 14: 1-11.

Keywords: magnetic resonance imaging, turbulence

\section{INTRODUCTION}

In general, arterial flow is laminar but becomes turbulent distal to a stenosis, even for a relatively moderate degree of stenosis. The effect of laminar flow on magnetic resonance (MR) images is well studied [1], but variable signal, typical noted in the presence of vascular stenoses, degrades the depiction of the vascular lumen, and reduces the diagnostic value of MRA studies. Assuming that each spin moves randomly during the application of magnetic gradient, Carr \& Purcell[2] arrived at a conclusion that the signal loss is proportional to $e^{-\psi^{2}, 2}$ where $\phi$ is the phase fluctuation [3]. This diffusive mechanism resulting from random motions, as suggested by Kuethe[4], can not describe the even echo rephasing.

Several different models $[3,5,6]$ have been proposed and the general expression of phase fluctuation

Received: Oct. 8, 2001; accepted: Jan. 15, 2002 Correspondence: Liang-Der Jou, VA Medical Center (114D) Department of Radiology

University of California at San Francisco,

San Francisco, CA 94121, USA in a spin echo sequence can be written as

$$
\left\langle\phi^{2}\right\rangle=2 \gamma^{2} G^{2}\left\langle u^{\prime 2}\right\rangle f\left(T_{0}, \tau\right),
$$

where $T_{0}$ is the flow correlation time and $\tau$ is half the duration of a bipolar gradient. In this expression, the phase $\phi$ is proportional to $u^{\prime}$, the velocity fluctuation in the direction of magnetic gradient; and $\gamma G_{x}$, the product of the gyromagnetic ratio and the magnetic gradient. However, after substituting $M=M_{x}$ $+i M_{y}=M_{1} e^{-1 \phi}$ into the Bloch equation for the transverse magnetization, one has

$$
\frac{\partial \phi}{\partial t}+\boldsymbol{u} \cdot \nabla \phi=-\gamma G \cdot \boldsymbol{x} .
$$

Clearly the phase does not vary with the velocity scale because of nonlinear nature of the convection term. Therefore, Eqn. (1) does not fully describe the signal loss due to turbulence.In fact, Eqn. (1) is a special solution of Eqn. (2) when the convection term is neglected or $\tau \ll T_{0}[3]$.

NMR signal loss in a homogeneous isotropic turbulence has been investigated.[3,7-8] In their studies, the Lagrangian time scale is approximated to the 
Eulerian time scale[3-4], and this approximation is questionable. There is a clear indication that the Lagrangian time scales are larger than the Eulerian time scales $[9,10]$ since the velocity of the same particle correlates longer than the velocity of two particles which occupy the same location but at different time. Despite of the approximation of two time scales (Eulerian and Lagrangian), Eqn. (1) provides very good agreements with the experiments,[4] due to the fact that the turbulent time scale is much larger than the duration of magnetic gradient. When $\tau / T_{0}$ is much smaller than 1 for a bipolar gradient, Eqn. (1) reduces to an expression that is independent of $\tau / T_{0}$. Therefore, the choice of turbulent time scale (Lagrangian or Eulerian) is not important for a bipolar gradient; however, for a flow compensated gradient, $\left\langle\phi^{2}\right\rangle$ is proportional to $\tau / T_{0}$, and the signal loss was not observed until at a Reynolds number that is not in the physiological range [11].

The effect of turbulence on MR images were investigated by Evans et al.[12], Urchuk et al.[13] and Oshinski et al.[14] It was found that the decrease of signal intensity due to turbulence was negligible in fully developed pipe flow except at a very high Reynolds number[12]; however, a dark region distal to a stenosis was observed for the Reynolds number as low as 600 . Note that flow compensations in both sliceselective and readout directions were used in the experiments. The decrease of signal intensity results from the phase fluctuation varying in each phase encoding step due to the velocity fluctuation. In the literature, the shear flow effect has not been addressed.

In this paper the Bloch equation is examined and a different way to describe the signal loss for turbulent flow is proposed. Specifically, a fully developed pipe flow, where the statistics of flow characteristics is available, is considered and the effect of flow shear on the signal loss is discussed.

\section{THEORY}

Introduce $\phi=\phi_{u}+\phi_{s}$ where $\phi_{s}=-x \cdot \int_{0}^{t} \gamma G d t$ and $\phi_{s}$ is the phase acquired by stationary tissue;
Eqn. (2) can then be written as

$\frac{\partial \phi_{u}}{\partial t}+u \cdot \nabla \phi_{u}=u \cdot \int_{0}^{t} \gamma G d t$

Note that all the variables in the equation are in terms of Eulerian variables and do not rely on the particle's location.

For simplicity, consider a two dimensional flow. Substituting $u=\bar{u}+\epsilon u^{\prime}, v=\bar{v}+\epsilon v^{\prime}$ and $\phi_{u}=\phi_{0}+\epsilon \phi_{1}+\epsilon^{2} \phi_{2}+\cdots$ into Eqn. (3) and separating the terms with different orders of $\epsilon$, one has

$$
\begin{aligned}
& \frac{\partial \phi_{0}}{\partial t}+\bar{u} \frac{\partial \phi_{0}}{\partial x}+\bar{v} \frac{\partial \phi_{0}}{\partial y}=\bar{u} \int^{\prime} \gamma G_{x} d t+\bar{v} \int^{\prime} \gamma G_{y} d t \\
& \frac{\partial \phi_{1}}{\partial t}+\bar{u} \frac{\partial \phi_{1}}{\partial x}+\bar{v} \frac{\partial \phi_{1}}{\partial y}=\bar{u} \int^{\prime} \gamma G_{x} d t+v^{\prime} \int^{\prime} \gamma G_{y} d t-u^{\prime} \frac{\partial \phi_{0}}{\partial x}-v^{\prime} \frac{\partial \phi_{0}}{\partial y} \\
& \frac{\partial \phi_{2}}{\partial t}+\bar{u} \frac{\partial \phi_{2}}{\partial x}+\bar{v} \frac{\partial \phi_{2}}{\partial y}=-u^{\prime} \frac{\partial \phi_{1}}{\partial y}-v \frac{\partial \phi_{1}}{\partial y}
\end{aligned}
$$

For flow in a long straight channel, $\bar{u}=\bar{u}(y)$ and $\bar{v}=0$. Also, the bipolar gradient (as shown in Figure $1 b$ ) is assumed to be in the direction of flow ( $x$ direction) and its duration is $2 \tau$. Eqn. (4) yields $\phi_{0}=\gamma G_{x} \bar{u} t^{2} / 2$ for $0<t<\tau$ and $-\gamma G_{x} \bar{u}\left[(2 \tau-t)^{2}\right.$ $\left.12-\tau^{2}\right]$ for $\tau<t<2 \tau$. Assuming $z=x-\bar{u}(y) t$, one can transform $\phi_{1}(x, y, t)$ into a function of $z, y$ and $\mathrm{t}$ and rewrite Eqn. (5) as

$$
\frac{\partial \phi_{1}(x, y, t)}{\partial t}+\bar{u} \frac{\partial \phi_{1}(x, y, t)}{\partial x}=\frac{\partial \phi_{1}(z, y, t)}{\partial t}
$$

That is, the derivative is now along the mean particle path rather than the true particle path. By doing so, one can avoid the errors generated from tracking individual particle. Note that the coordinate transformation does not affect $u^{\prime}$ and $v^{\prime}$, and all the terms on the right hand side of Eqns. (5) and (6) remain the same. Another reason that the current approach is preferred over the true particle path[3] is that only the phase fluctuation at a particular location, not for a specific particle, need to be determined; especially for shear flow, the signal at a specific location may originate from particles that are from different locations each time the

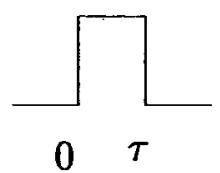

(a)

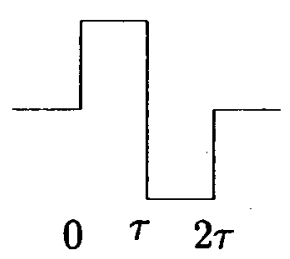

(b)

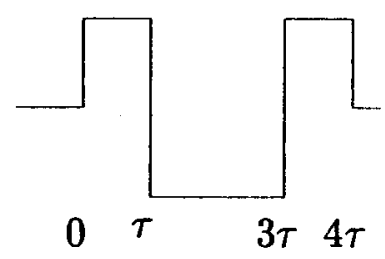

(c)

Fig. 1. (a) Single pulse gradient, (b) bipolar gradient and (c)a simple flow compensated gradient. 
phase encoding is applied. In addition, measuring the Lagrangian correlation experimentally is extremely difficult. Calculating the Lagrangian correlation numerically is also difficult and the numerical errors generated from tracking particles in direct numerical simulation for turbulence has been discussed by Yeung \& Pope[15]. Integrating Eqn. (5) yields

$$
\begin{aligned}
& \phi_{1}(t=2 \tau)=-\frac{\gamma G_{x}}{2} \frac{d \bar{u}}{d y} \int_{1}^{\tau}\left[v_{1}^{\prime} t^{2}-v_{2 t-t^{\prime}}^{\prime}\left(t^{2}-2 \tau^{2}\right)\right] d t \\
& +\gamma G_{x} \int_{0}^{\tau}\left[u_{t}^{\prime}+u_{2 r-t}^{\prime}\right] t d t .
\end{aligned}
$$

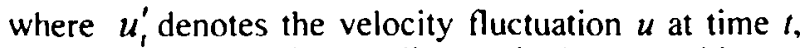
and the same notation applies to $v^{\prime}$. The ensemble average of phase $\phi_{1}$ at $t=2 \tau$ can be expressed as

$$
\begin{aligned}
& \frac{<\phi_{1}^{2}>}{\left(\gamma G_{x}\right)^{2}}=\frac{1}{2}\left(\frac{d \bar{u}}{d y}\right)^{2}<v^{\prime 2}>\int_{0} \int\left\{\left[R_{w}^{\prime-t}-R_{w}^{2 r-t-r^{\prime}}\right]\right. \\
& \left.\left[t^{2} t^{\prime 2}-\tau^{2}\left(t^{2}+t^{\prime 2}\right)\right]+2 \tau^{4} R_{v v}^{t-t^{\prime}}\right\} d t d t^{\prime} \\
& +2<u^{\prime 2}>\oint \oint\left[R_{u \nu}^{\prime-1}+R_{u \nu}^{2 t-1 \cdot t^{\prime}}\right] \| t^{\prime} d t d t^{\prime} \\
& -\left(\frac{d \bar{u}}{d y}\right) \int_{0} \int_{0}\left[<u_{t}^{\prime} v_{t}^{\prime}\right\rangle t^{\prime 2}-\left\langle u_{t}^{\prime} v_{2 \gamma-t^{\prime}}^{\prime}\right\rangle \\
& \left(t^{\prime 2}-2 \tau^{2}\right)+<u_{2 r}^{\prime}, v_{t}^{\prime}>t^{\prime 2}-<u_{2 r}^{\prime}, v_{2 r-t^{\prime}}^{\prime}> \\
& \left.\left(t^{\prime 2}-2 \tau^{2}\right)\right] t d t d t^{\prime} \\
& =T_{v}^{6}\left(\frac{d \bar{u}}{d y}\right)^{2}<v^{\prime 2}>f_{v}^{h p}\left(\tau, T_{v}\right) \\
& +<u^{\prime 2}>T_{u}^{4} f_{u}^{h p}\left(\tau, T_{u}\right) \\
& -\left(\frac{d \bar{u}}{d y}\right)<u^{\prime} v^{\prime}>T_{w}^{s} f_{w}^{p r}\left(\tau, T_{w}\right)
\end{aligned}
$$

where $R_{u u}$ and $R_{v v}$ are two-point correlation functions and superscript $b p$ denotes the bipolar gradient. The definitions of $R_{u u}$ and $R_{v}$ are $R_{u s}^{\prime \prime}=\left\langle u_{t}^{\prime} u_{t^{\prime}}^{\prime}\right\rangle /\left\langle u^{\prime 2}\right\rangle$, and $R_{\mathrm{v}}^{\prime \prime}=\left\langle v_{t}^{\prime} v_{t}^{\prime}>/<v^{\prime 2}>\right.$.

Note that $u_{1}^{\prime} u_{i}^{\prime}=u^{\prime}\left(z_{1}, t\right) u^{\prime}\left(z_{2}, t^{\prime}\right)$ and $z_{1}-z_{2}=$. $\bar{u}\left(t-t^{\prime}\right)$ In the following discussion, the first term of Eqn. (9) is denoted as VFT, the second term as UFT and the last term as RST. Note that $\left\langle u_{t}^{\prime} v_{r}^{\prime}\right\rangle$ is similar to the Reynolds stress, $-\left\langle u_{1}^{\prime} v_{1}^{\prime}\right\rangle$,except that there is a time delay between $u^{\prime}$ and $v^{\prime}$. In addition, $\left\langle u_{t}^{\prime} v_{r^{\prime}}^{\prime}\right\rangle$ and $\left\langle u_{t}^{\prime}, v_{t}\right\rangle$ are not the same. The flow conditions that correspond to each term in Eqn. (9) are illustrated in Figure 2. It shows that the random motions in the $y\left(v^{\prime}\right)$ and $x\left(u^{\prime}\right)$ direction are the causes of VFT and UFT, while a vortical motion (Fig. 2c) can contribute to all three terms in Eqn. (9). There is no single type of motion that will generate RST without causing UFT and VFT, and VFT and RST represent the gradient transport in the presence of flow shear. With $R_{u \nu}^{\prime}=e^{\prime \prime T_{0}}$ and either $v^{\prime}=0$ or no flow shear, Eqn. (9) reduces to Eqn. (1) and has the same expression for $f\left(T_{11}, \tau\right)$ as that given by Gao \& Gore [3]. Similarly, one can obtain phase fluctuation for a single unipolar gradient and a flow-compensated gradient, and the results are included in Appendix 2 and 3.

An alternative way of deriving Eqn. (9) is to follow the Lagrangian approach[3]. The velocity of a particle $\xi$ at time $t$ is written as

$$
u=\bar{u}_{12}+u_{1}^{\prime}-\frac{d \bar{u}}{d y} \int^{2} v_{t}^{\prime} \cdot d t^{\prime}
$$

in a shear flow environment. The displacement of the particle $\xi$ in the $x$-direction between time $t_{1}$ and $t_{2}$ is

$$
x_{t,}-x_{11}=\int_{1}^{2} u_{t} d t=\int_{1}^{2}\left(\bar{u}_{12}+u_{1}^{\prime}-\frac{d \bar{u}}{d y} \int^{2} v_{1}^{\prime} \cdot d t^{\prime}\right) d t .
$$

The ensemble average of displacement for an interval $t$ then yields to

$$
\begin{aligned}
& \left\langle\left(x_{i_{1}+2}-x_{i_{i}}\right)\left(x_{i_{1}+5}-x_{i_{i}}\right)\right\rangle \\
& =\bar{u}^{2} t^{2}+2\left\langle u^{\prime 2}>\int_{t_{1}}^{\tau}\left(\tau-t^{\prime}\right) R_{u, t}^{\prime} d t^{\prime}\right. \\
& +\frac{1}{3}\left(\frac{d \bar{u}}{d y}\right)^{2}<v^{\prime 2}>\int_{n}^{\prime}\left(t^{3}-\pi^{2}+2 \tau^{\prime}\right) R_{n}^{\prime} d t \\
& -2 \frac{d \bar{u}}{d y} \int_{0}^{t} \int_{0}^{t}<u_{t_{1}+,}^{\prime}, v_{t_{1},+}^{\prime}>t d t d t^{\prime}
\end{aligned}
$$

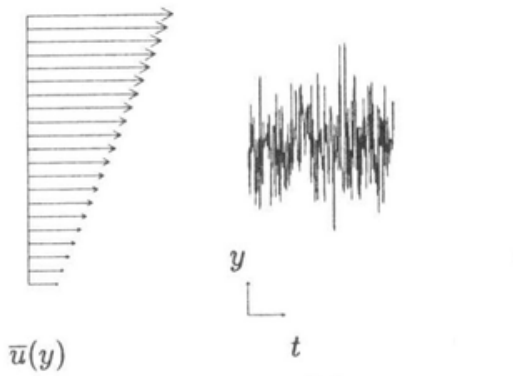

(a)

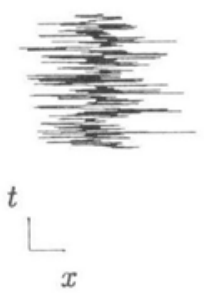

(b)

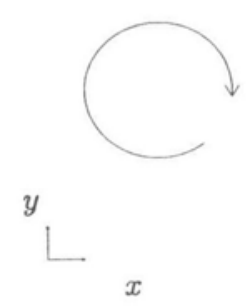

(c)

Fig. 2. The mechanism of signal loss in turbulence. Random motion in y-direction (a) and x-direction (b). Vortical motion or rotation (c). 
With

$<\phi^{2}>=\gamma^{2} G_{x}^{2} \int_{1}\left[<\left(x_{t_{1}+\tau}-x_{t_{1}}\right)\left(x_{t_{2}+\tau}-x_{t_{1}}\right)>d t_{1} d t_{2}-\phi_{0}^{2}\right.$ and following the same procedure as in Gao \& Gore[3], one would arrive at an expression similar to Eqn. (9).

If the signal is not acquired immediately after the bipolar gradient and there is a time delay between the bipolar gradient and data acquisition, additional dephasing will occur. Suppose $t_{e}$ is the time delay and $\phi_{e}$ is the phase, then

$\phi_{e}=\phi_{1}+g_{x} \tau^{2} \frac{d u}{d y} \int_{r}^{r_{r}+2 x} v_{1}^{\prime} d t$.

The ensemble average of $\phi_{e}^{2}$ is

$<\phi_{c}^{2}>=<\phi_{1}^{2}>+\gamma^{2} G_{x}^{2} \tau^{2}<v^{\prime 2}>\left(\frac{d \bar{u}}{d y}\right)^{2} \int_{t}^{+2 \pi} \int R_{v v}^{\prime-t^{\prime}} d t d t^{\prime}$

$+\gamma^{2} G_{\gamma}^{2} \tau^{2}\left(\frac{d \bar{u}}{d y}\right)^{2} \int_{0}^{0} \int_{,}\left[R_{v v}^{\prime+2 \tau-1} t^{2}-R_{v v}^{\prime+1}\left(t^{2}-2 \tau^{2}\right)\right] d t d t^{\prime}$

$-2 \gamma^{2} G_{x}^{2} \tau^{2} \frac{d \bar{u}}{d y} \int_{0}^{*} \int_{b}\left(<v_{r^{\prime}+2 r}^{\prime} u_{r}^{\prime}>+<v_{i+2 r}^{\prime} u_{2 r-1}^{\prime}>\right) t d t d t^{\prime}$

The relation implies that the signal will decrease even after the magnetic gradient is turned off. The fluctuation of velocity in the cross-stream direction is the cause of the dephasing. This dephasing was not predicted in the previous studies, in which there was no flow shear.[3,8] In addition, the signal decays with $e^{-t,}$ and $e^{-t_{*}^{2}}$, compared with $e^{-t_{*}}$, the decay rate when $\int G_{x} d t \neq 0$. [8] as

The modified Bloch equation[4] can be written

$$
\frac{\partial M}{\partial t}+u \cdot \nabla M=-i \gamma G \cdot x M+D, \nabla^{2} M
$$

where $M$ is the transverse magnetization. Let $M=M_{x}+i M_{v}=M_{1} e^{i \phi}$ then one has

$\frac{\partial \phi}{\partial t}+u \cdot \nabla \phi=-\gamma G \cdot x+D_{t} \nabla^{2} \phi+i D_{\imath}(\nabla \phi) \cdot(\nabla \phi)$.

Without the last term, the phase will simply diffuse and will not generate any signal loss. Introduce $\phi=\phi_{s}+\phi_{y}+i \phi_{,}$, then the equation becomes

$\frac{\partial \phi_{y}}{\partial t}+u \cdot \nabla \phi_{r}=u \cdot \int \gamma G d t^{\prime}+D, \nabla^{2} \phi_{r}-2 D, \nabla \phi_{r} \cdot\left(\nabla \phi_{s}+\nabla \phi_{r}\right)$

$\frac{\partial \phi_{1}}{\partial t}+u \cdot \nabla \phi_{i}=D_{1} \nabla^{2} \phi_{1}+D_{1}\left(\nabla \phi_{s}+\nabla \phi_{r}\right) \cdot\left(\nabla \phi_{s}+\nabla \phi_{r}\right)$

where $\phi_{s}=-x \cdot \int \gamma G d t^{\prime}$. The decrease of signal is determined by the imaginary part of $\phi, \phi_{i}$. The nonlinearity in Eqns. (12-13) illustrates that the phase $\phi$ can not possibly be a linear function of $\gamma^{2} G_{x}^{2}$.

Suppose $u=(\bar{u}, \bar{v})=(\vec{u}, 0)$, , where $\bar{u}$ is a con- stant, then $\phi$ and $\phi$ are not functions of position and the equation for $\phi_{r}$ can be simplified to

$$
\frac{d \phi_{1}}{d t}=D_{i}\left(\nabla \phi_{s}\right) \cdot\left(\nabla \phi_{s}\right)
$$

The integration of $\phi_{i}$ yields $\phi_{i}=\gamma^{2} G_{x}^{2} D_{i} \tau^{3} / 3$. Comparing with $\left\langle\phi_{1}^{2}\right\rangle 2$ for the limiting case of $\tau \gg T_{u}, T_{v}$ and $T_{u v}$ for a simple gradient pulse in Appendix 2, one can obtain $D_{1}=<u^{\prime 2}>T_{*}$. This result holds for the bipolar and flow-compensated gradients as well. For positive $D_{t}$, the last term on the right hand side of Eqn. (13) contributes to the loss of the signal, whereas the first term tends to balance the loss spatially, and thus decreases the loss. In a turbulent pipe flow, the signal loss is the greatest near the wall, at which the diffusion effect of $\phi$, can be found.

Consider a case of simple shear flow, where $u$ $=A y$ and $\mathrm{A}$ is a constant. The solutions for $\phi_{r}$ and $\phi$ at $t=\tau$ are

$$
\phi_{r}=\frac{1}{2} \gamma G_{x} \tau^{2} A y
$$

and

$\phi_{1}=\frac{1}{20} D_{1} \gamma^{2} G_{x}^{2} A^{2} \tau^{3}+\frac{1}{3} D_{1} \gamma^{2} G_{x}^{2} \tau^{3}$

Comparing $\phi$, with $\left\langle\phi_{1}^{2}>/ 2\right.$ in Appendix 2, one then have

$$
D_{1}=\frac{\left\langle u^{\prime 2}>T_{u}+\frac{3}{20} A^{2}<v^{\prime 2}>\tau^{2} T_{v}-\frac{3}{8} A<u^{\prime} v^{\prime}\right\rangle \tau T_{u v}}{1+\frac{3}{20} A^{2} \tau^{2}}
$$

for $\tau \gg T_{v}, T_{\text {v }}$ and $T_{v v}$. Therefore, $D_{t}$ is not an universal constant for all flows. For $\tau=1 \mathrm{~ms}$ and $\frac{d \bar{u}}{d y}=A<<1000 s^{-1}, D_{t}=<u^{\prime 2}>T_{\prime \prime}$ is still a very good approximation. The velocity gradient near the wall is around $1000 \mathrm{~s}^{-1}$ at the Reynolds number of 7200 in a pipe of $1 \mathrm{~cm}$-diameter. For any other flows such that $u=u(y), \phi$, and $\phi$, are functions of $y$. Then one can not simplify the equations, and $D_{t}$ can not be simply represented as $\left\langle u^{\prime 2}>T_{u}\right.$. Therefore, the choice of $D_{t}$ depends on the type of flows under consideration.

In fact, $D_{1}$ arises from the need to model the correlation of the fluctuations of velocity and magnetization; the fluctuation is treated as a diffusive flux:

$$
\nabla \cdot\left\langle u^{\prime} M^{\prime}\right\rangle=-D_{1} \nabla \cdot \nabla M
$$

With the Bloch equation:

$$
\frac{\partial M}{\partial t}+\nabla \cdot(u M)=-i \gamma G \cdot x M,
$$




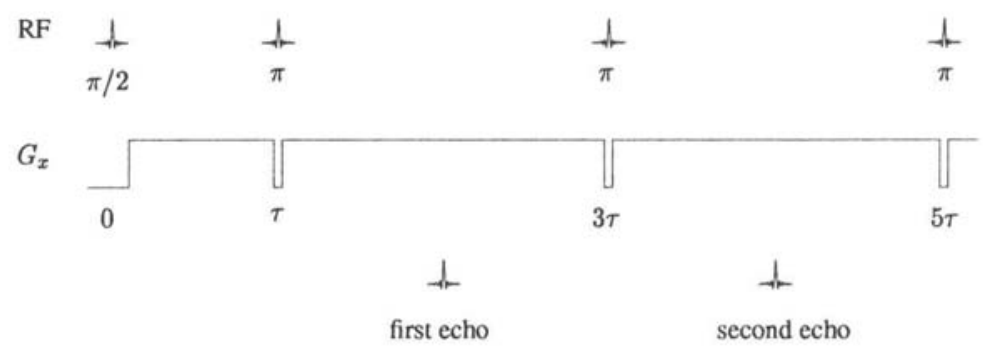

Fig. 3. A spin echo sequence.

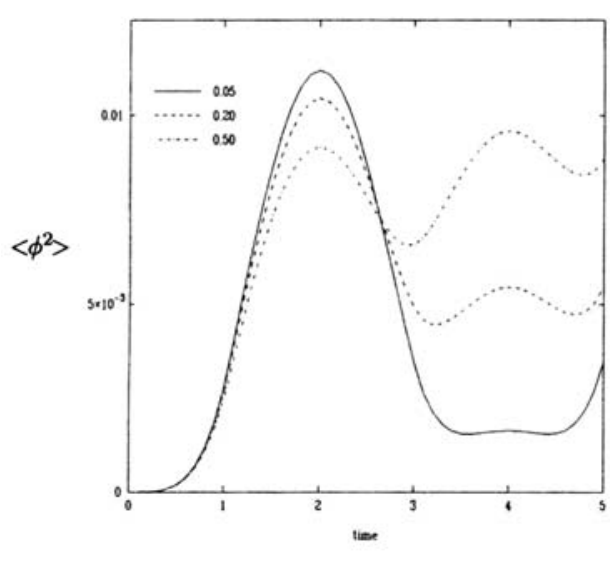

(a)

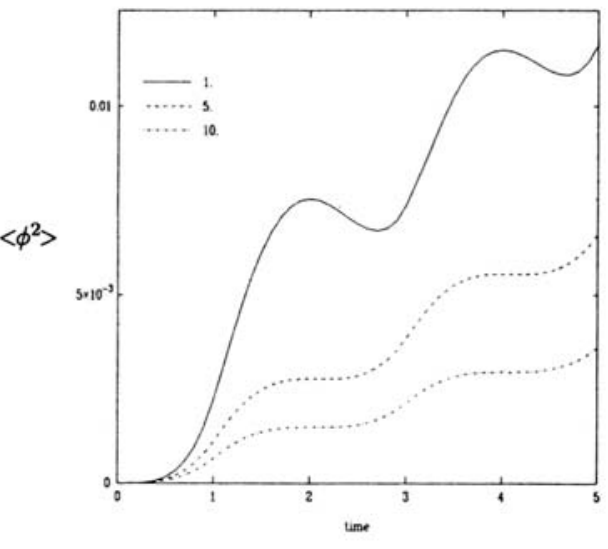

(b)

Fig. 4. Phase variation $\left(<\phi^{2}>\right)$ for different $\tau / T_{10}(0.05,0.2,0.5$ in (a) and $1,5,10$ in (b)) in a spin echo sequence. In the plot, $\tau$ is fixed, and time is normalized by $\tau$

and $M=M+M^{\prime}$ and $u=u+u^{\prime}$, the ensemble average of the resulting equation becomes

$$
\frac{\partial M}{\partial t}+\nabla \cdot\left(u M+<u^{\prime} M^{\prime}>\right)=-i \gamma G \cdot x M
$$

This equation is exactly the same as the modified Bloch equation if Eqn. (14) holds. In deriving the relation $D_{1}=<u^{\prime 2}>T_{u}$, one has to assume that $\tau \gg T_{u}, T_{v}$ and $T_{u v}$, which is not true for most of cases.

\section{RESULTS}

\subsection{A spin echo sequence}

Consider a spin echo sequence as shown in Fig. 3. Following the same procedure as described before, one can have a relation for the ensemble average of the phase fluctuation at the first echo $(t=2 \tau)$ and the second echo $(t=4 \tau)$. These relations reduce to the results in Gao \& Gore[3] when $\frac{d \bar{u}}{d y} \tau<1$.

The change of $\left\langle\phi_{1}^{2}\right\rangle$ with respect to time in the absence of flow shear is shown in Fig. 4, in which $\tau$ is fixed and time is normalized by $\tau$. As shown in Fig. 4a, for small $\tau / T_{0}$ the periods of time $(2 \tau<t<3 \tau$ and $4 \tau<t<4.6 \tau)$ that correspond to the decreasing of $\left\langle\phi_{1}^{2}\right\rangle$ has negative eddy diffusivity. The negative eddy diffusivity causes the rephasing of spins. In turbulent theory, energy tranfers from low frequency to high frequency and then dissipates at small (Kolmogorov) scale. Occasionally energy transfers in the opposite direction, and it is called backscatter. Similarly, negative $D_{t}$ corresponds to the case where the phase becomes correlated again.

As $\tau / T_{0}$ increases above 5 , the rephasing is unlikely to occur. Eventually, the phase fluctuation will grow as $\left\langle u^{\prime 2}\right\rangle \tau^{3} T_{0}$, and $D_{t}$ is dominated by the flow rather than by the gradient sequences because it consists of several turn-over time $\left(T_{0}\right)$ in one pulse gradient. The limiting value of $D_{t}$ is $\left\langle u^{\prime 2}>T_{n}\right.$. The assumption of exponential decay of $D_{t}$ does not describe the rephasing properly, either[4]. Since $\nabla \phi_{s} \cdot \nabla \phi_{1}=0$ at the time of the first and second echoes, $\partial \phi, / \partial t$, which is proportional to the eddy diffusivity, is close to zero.

One can conclude that when $\tau<<T_{0}$ the signal loss is pulse sequence-dominated. When $\tau \gg T_{10}$, the signal loss is determined by the flow structure, and is purely diffusive with no rephasing. Because of the diffusive nature associated with large $\tau / T_{n}$, the signal 


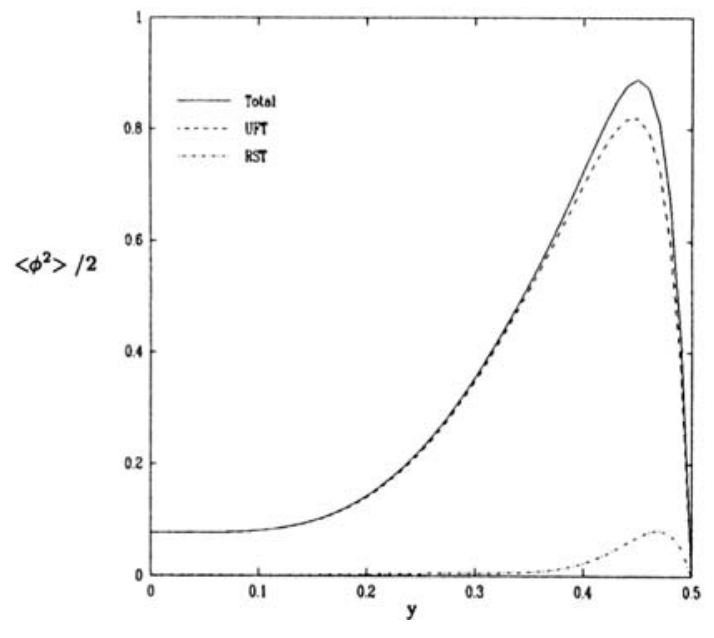

Fig.5 Calculated dephasing budget for the bipolar gradient in turbulent pipe flow.

drops less than for larger $\tau / T_{0}$ at the first echo and the second echo. It can be interpreted as follows. When $T_{0} \gg \tau$ the velocity $u^{\prime}$ fluctuates less and is likely to be in the same direction during the time that the magnetic gradient is applied. Therefore, the fluctuating phase accumulates at the first echo, but is compensated at the second echo. The velocity $u^{\prime}$ is truly random during the application of magnetic gradient when $T_{0} \ll \tau$. Thus, the phase will not accumulate as great as that when the velocity is correlated, and the flow effect is less compensated at the second echo as shown in Figure $4 b$.

\subsection{Turbulent pipe flow}

Data in Table 1 is obtained from Eggels et al.[16] and Westerweel et al.[17], where two-point correlation functions were calculated by direct numerical simulation for turbulent pipe flow. $T_{u}$ and $T_{v}$ are estimated with the relations $T_{v}=L_{v} / \bar{u}$ and $T_{v}=L_{v} / \bar{u}$. [17] The rationale of these relations is based on that the twopoint velocity correlation has a maximum at $x=\bar{u} \tau$ where $x$ is the separation between two points, $\tau$ the time delay and $\bar{u}$ the average velocity.[9] It has been demonstrated that $L_{v}$ is independent of shear rate, while $L_{u}$ increases with the increase of the shear rate. [17] All the length scales are normalized by the diameter and velocity by the friction velocity. For the maximum velocity of $72 \mathrm{~cm} / \mathrm{s}$ and the diameter $10 \mathrm{~mm}$, the friction velocity is $3.7 \mathrm{~cm} / \mathrm{s}$. For a diameter of $30 \mathrm{~mm}$, the maximum velocity is $24 \mathrm{~cm} / \mathrm{s}$ and the friction velocity is $1.2 \mathrm{~cm} / \mathrm{s}$. Eqn. (9) suggests that the larger the diameter of the pipe, the less the signal loss for the same Reynolds number.[14]

Note that the estimation of time scale is $140 \mathrm{~ms}$ for $T_{u}$ near the wall $(y=0.487)$ and $20 \mathrm{~ms}$ at the center $(y=0.008)$, while the estimation by Gao \& Gore[3] was $200 \mathrm{~ms}$. The time scale $T_{v}$ is $10 \mathrm{~ms}$ at $\mathrm{y}=0.008$ and $42 \mathrm{~ms}$ at $y=0.487$. Since few experimental measurement of the time scale $T_{u v}$ is available, the importance of the cross-correlation term $\left\langle u^{\prime} v^{\prime}\right\rangle$ can not be determined accurately. The significance of the Reynolds stress and its role in turbulent momentum transport have been discussed by Bernard et al.[19] It is suggested that the inertial effect (deceleration/acceleration) contributes to most of $\left\langle u^{\prime} v^{\prime}\right\rangle$ The estimation of $T_{u v}$ is $450 \mathrm{~ms}$ near the wall at a slightly higher Reynolds number of 8,900 by Chevrin et al. [20] These estimations of time scales indicate that $T_{u}, T_{v}$ and $T_{z y}$ are at the same order of magnitude and are larger than the MR time scale, $\tau$. Assuming that $T_{u v} \gg \tau$, one can make an approximation that $\left.\left\langle u_{i}^{\prime} v_{f}^{\prime}\right\rangle \approx \Delta u_{1}^{\prime} v_{i}^{\prime}\right\rangle$ and then $f_{u v}^{b p}\left(\tau, T_{u v}\right) \approx 2\left(\tau / T_{u v}\right)[5]$ Since $T_{u v}$ does not appear in $R S T$ explicitly if $T_{u v} \gg \tau$, the choice of $T_{u v}$ does not affect the final result.

Although the data in Table 1 are for the fullydeveloped pipe flow [16-17], the qualitative information can be extracted by applying these data to our two-dimensional channel flow model. With these data, one can estimate the contribution for each term in Egn. (9) and they are shown in Fig. 5-6. The curves are obtained by fitting the data at 4 locations $(y=0.008$, $0.247,0.451$ and 0.487 ) with zero slope at $y=0$ and zero phase fluctuation at $y=0.5 .1 \mathrm{G} / \mathrm{cm}$ is used for the magnetic gradient in these estimates. One can observe that the signal loss due to the velocity fluctuation $\left\langle u^{\prime 2}\right\rangle$ has the same order of magnitude across the diameter but is significantly larger near the wall, and the total signal loss is the lowest for the flowcompensated pulse sequence in Fig. 7. The effect of the velocity fluctuation $\left\langle v^{\prime 2}\right\rangle$ is three to four times larger for the flow-compensated gradient than that in the bipolar gradient. The signal loss caused by $\left\langle u^{\prime} v^{\prime}\right\rangle$ is still less than that due to the velocity fluctuation $u^{\prime}$ for the bipolar gradient. Since we have assumed that $T_{u v} \gg \tau$, RST $=0$ for the flowcompensated gradient, i.e., the cross-correlation of the velocity results in signal loss for the bipolar gradient while causing no signal loss in the flow-compensated pulse.

A comparison of different gradients is shown in Fig. 7. The signal loss for the flow-compensated gradient is an order of magnitude smaller than that for the bipolar gradient. The signal loss in turbulence is the greatest for a bipolar gradient, and the greatest signal loss is around 0.05 diameters away from the wall, which corresponds to the location of maximum $\left\langle u^{\prime 2}>\right.$. [17]

\subsection{Turbulent mixing layer}

Another example is the mixing layer generated by the flow through a moderate stenosis[20]. When the 
stenosis is serious, the flow becomes a round jet and the appearance of MR image is different from that of a moderate stenosis. A large velocity gradient across this layer, which separates the fast fluid and the stagnant fluid, will amplify the effect of shear flow on the NMR signal loss. At moderate Reynolds number $(\approx 3000)$, a transition region of one diameter long was observed. Distal to that area, the flow becomes turbulence.[21] However, the transition region, which is unstable but laminar, appeared dark due to the presence of high flow shear.[22] Since flow phantom is exposed to high frequency noises in the scanner, compared with the controlled environment in fluid dynamics experiment, the critical Reynolds number was much lower in phantom study.

Assuming $T<<T_{u}, T_{v}$ and $T_{u v}$, the ensemble average of the phase fluctuation for a flow compensated gradient can be written as (in Appendix 3)

$$
\begin{aligned}
& \frac{<\phi_{1}^{2}>}{2}=\frac{23}{15} \gamma^{2} G_{x}<u^{\prime 2}>\frac{\tau^{5}}{T_{u}}+ \\
& 2 \gamma^{2} G_{x}^{2}\left(\frac{d \bar{u}}{d y}\right)^{2}<v^{\prime 2}>\tau^{6}
\end{aligned}
$$

A plot of $\left\langle\phi_{1}^{2}\right\rangle / 2$ at 0.25 diameter distal to a $50 \%$ axisymmetric sudden expansion is shown in Fig. 8 by fitting the experimental data[23] and assuming $\tau / T_{\star}=$ 0.1 . One can see that strong flow shear increases the dephasing and the signal loss. Unfortunately, $\left\langle u^{\prime 2}\right\rangle,\left\langle v^{\prime 2}\right\rangle$ and $\left\langle u^{\prime} v^{\prime}\right\rangle$ have the same type of distribution but with different magnitude, and it is unlikely to separate one from another experimentally.

Another factor that has not been addressed is the

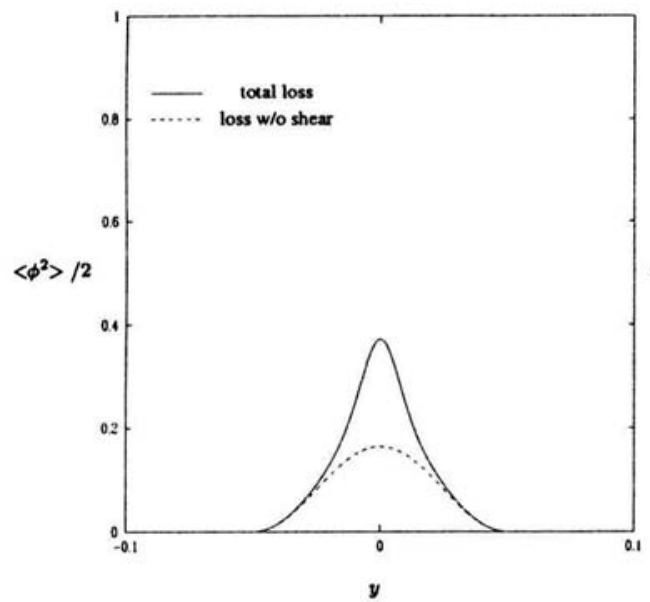

(a) intermittency in a post-stenotic jet. The distribution of intermittency is similar to the distribution of $\left\langle u^{\prime 2}\right\rangle$ and is 1 (fully turbulent) at the center of the mixing layer $(x=0)$. Therefore, the role of intermittency in NMR signal loss is still unknown. However, it is believed that the higher the intermittency, the larger the signal loss.

As shown in Figure 8, the effect of flow shear can increase the signal loss by $25 \%$ for a bipolar gradient and $50 \%$ for a flow-compensated gradient. The total amount of the signal losses for a bipolar gradient is twice larger than that of a velocity-compensated gradient pulses. Also, the region given by Fig. $8 \mathrm{a}$ is narrower than that in Fig. 8 b.

\section{DISCUSSION}

An explicit expression for the MR signal in turbulence is derived. In addition to the velocity fluctuation, $\left\langle u^{\prime 2}\right\rangle$ and $\left\langle v^{\prime 2}\right\rangle$ the MR signal depends on $\left\langle u_{t}^{\prime} v_{,}^{\prime}\right\rangle$, a quantity that resembles the Reynolds stress. There are two major differences between the previous studies and present study. First, Gao \& Gore[3] have assumed that the Lagrangian time scale is equivalent to the Eulerian time scale, which is not true for inhomogeneous turbulence in incompressible flow. Second, the integration is along the particle path by Gao \& Gore[3], whereas the integration of Eqn. (7) is for an imaginary point $(z, y)$. Note that $(z, y)$ can be viewed as a particle moving at the mean velocity. Therefore, $R_{u u}, R_{v v}$ and $\left\langle u^{\prime} v^{\prime}\right\rangle$ are neither the Lagrangian correlations nor the Eulerian correlations; however, they are easier to measure than the Lagrangian correlation. Favre[9] has indicated that these types of correlation are the envelopes of double space-

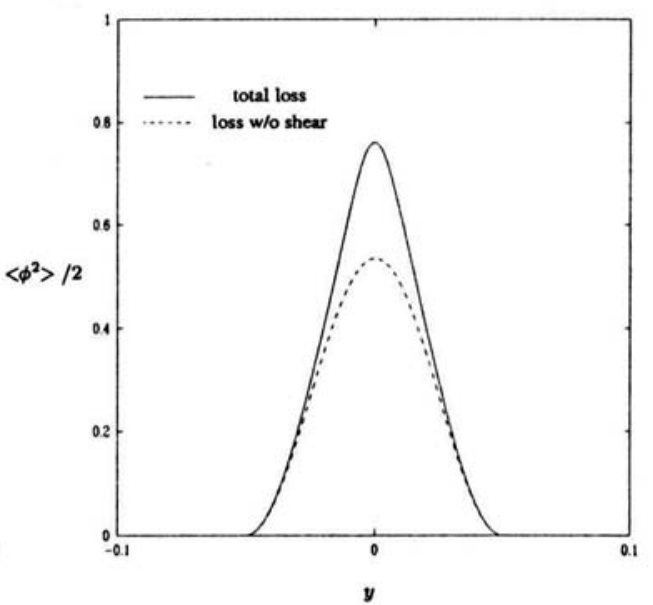

(b)

Fig.6 Calculated dephasing budget for the flow compensated gradient in turbulent pipe flow. 


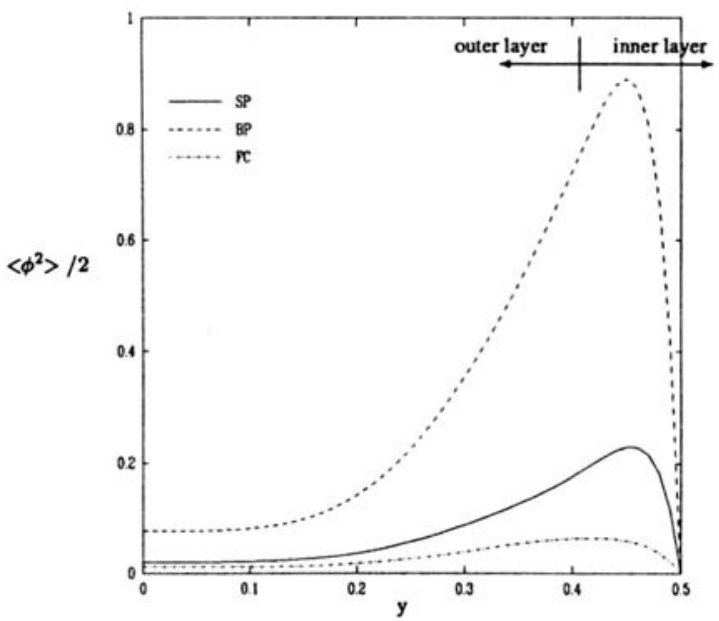

Fig. 7 Comparison of dephasing budget for three gradient pulses in turbulent pipe flow. SP: single pulse; BP: bipolar gradient; FC: flow compensated gradient.

time velocity correlations, and thus their integral time scales are longer. Unlike previous model, the current theory is valid for the fully developed turbulence in a straight pipe; the current model also gives a spatial variation of the signal and provides a theoretical mechanism that the cross-stream velocity fluctuation can cause the loss of signal in turbulent shear flow. The decrease of MR signal is dominated by $<u^{\prime 2}>$ near the center of the pipe, where $|d \bar{u} / d y|$ is small, especially for the bipolar gradient, but the effects of $\left\langle v^{\prime 2}\right\rangle$ and $\left\langle u^{\prime} v^{\prime}\right\rangle$ are not negligible near the wall. Provided that the explicit expressions for $R_{u u}$, $R_{v v}$ and $\left\langle u^{\prime} v^{\prime}\right\rangle$ are available, the detailed signal loss in turbulent pipe flow can be calculated. From experimental data, [16-17] we have estimated the contribution from each term in Eqn. (9). UFT is the dominated factor for the signal loss in the bipolar gradient, while VFT is more than doubled in the flow-compensated pulse, and it contributes a significant amount of loss.

In a bipolar gradient pulse, the velocity $\bar{u}$ is encoded in the phase of magnetization, and thus the velocity fluctuation $u^{\prime}$ dominates the phase fluctuation; no velocity information is encoded in the phase of magnetization in a flow-compensated pulse. The phase fluctuation generated by the first half of the flowcompensated gradient is compensated by the second half of the gradient. The Reynolds stress $\left\langle u^{\prime} v^{\prime}\right\rangle$ provides signal loss in the bipolar gradient pulse, but is negligible in the flow-compensate gradient pulse; its effect is at least one order of magnitude smaller than that of $\left\langle u^{\prime 2}\right\rangle$. One can easily visualize that the velocity fluctuation $v^{\prime}$ in a shear flow causes the change of the velocity in the mean flow direction, and generates the phase fluctuation. The situation worsens in the near wall region, where the velocity gradient is extremely high.

Eqn. (15) is not a linear function of velocity.
Since both the velocity fluctuation[24] and the velocity gradient increase with the flow rate, the terms on the right hand side of Eqn. (15) will increase differently. For example, if the flow rate is doubled, VFT increases by a factor of 16 , while UFT increases by a factor of 8 ( $T_{u}$ is halved). For a stenosis of $86 \%$ the region of signal void is much greater at the Reynolds number of 3000 for $40 \%$ glycerol-water mixture than that at the Reynolds number of 5600 for water.[24] The former velocity is $180 \mathrm{~cm} / \mathrm{s}$ while the latter is only $80 \mathrm{~cm} / \mathrm{s}$. Hence, the effect of flow shear is accentuated at higher velocity, and VFT can not be neglected. At the same flow rate, the image of water exhibits much greater signal loss than that of glycerol-water mixture; however, it does not imply a linear relation between the Reynolds number and the signal loss.

Gatenby \& Gore[25] measured the characteristic time of the turbulent eddies using pulsed-gradient NMR. They concluded that the characteristic time at the center of the pipe was longer than that near the wall. Since the temporal resolution of their images was $250 \mathrm{~ms}$, any time scale smaller than $250 \mathrm{~ms}$ can not be resolved. In fact, Fung[18] found from the kinematic simulation of turbulence that the time scale at higher flow shear rate was larger than that in homogeneous turbulence, and this is against their conclusion.

From dimensional analysis of the Bloch equations, and the Navier-Stokes equations, $\left\langle\phi^{2}\right\rangle=\gamma^{2} G^{2}$ $\tau^{4}\left\langle u^{\prime 2}\right\rangle f(\operatorname{Re}, \bar{u} \tau / D)$ where $D$ is the diameter of the pipe. Note that turbulent intensity, $\left\langle u^{\prime 2}\right\rangle / \bar{u}^{2}$ is a function of $\operatorname{Re}[24]$ Experiments have shown that $f$ increases with the Reynolds number. The fact that the signal loss was not strictly a function of the Reynolds number does not exclude the use of similarity in flow phantom.[14] There are more than one dimensionless variables, and the Reynolds number is only one of them. The other two are the ratio of the MR time scale $(\tau)$ to the flow time scale $(\mathrm{D} / \bar{u})$ and the magnetic gradient parameter $(\gamma G \tau D)$. Matching only one or two of them will not give a conclusive results. $[11,14]$ As demonstrated in our analysis, MR signal loss depends not only on the characteristics of the flow but also on the ratio of the time scales and the magnetic gradient strength. If $f(\operatorname{Re}, \bar{u} \tau / D)=f(\operatorname{Re}) g(\bar{u} \tau / D)$, where $f(\mathrm{Re})=\left(u^{\prime} / \bar{u}\right)^{3}$ and $g(\overline{\mathrm{u}} \tau / \mathrm{D})=\overline{\mathrm{u}} \tau / \mathrm{D}$, then

$<\phi^{2}>\sim\left(\frac{u^{\prime 3} \tau}{D}\right) \frac{\tau^{2}}{D^{2}}=\left(u_{\tau}^{\prime}\right)^{2} \frac{\tau^{2}}{D^{2}}$.

in which $\gamma G \sim 1 /(\tau D)$ is used and $u_{\text {, }}^{\prime}$ is the velocity fluctuation over the duration $\tau$. [14] According to Eqn. (16), one can halve the mean velocity and the diameter of the pipe, and use water as media instead of glycerol, then $\left\langle\phi^{2}>\right.$ will be the same because of the same Reynolds number and turbulent intensity. Although the area of signal void distal to the stenosis will 


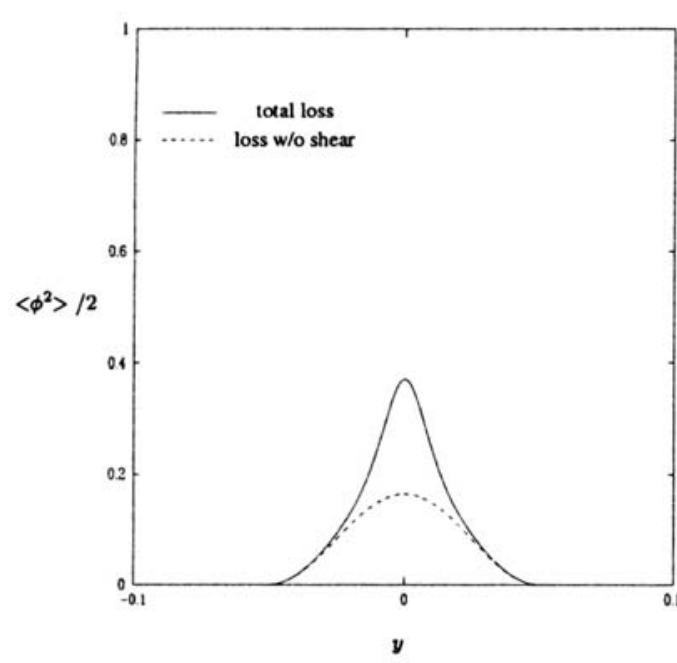

(a)

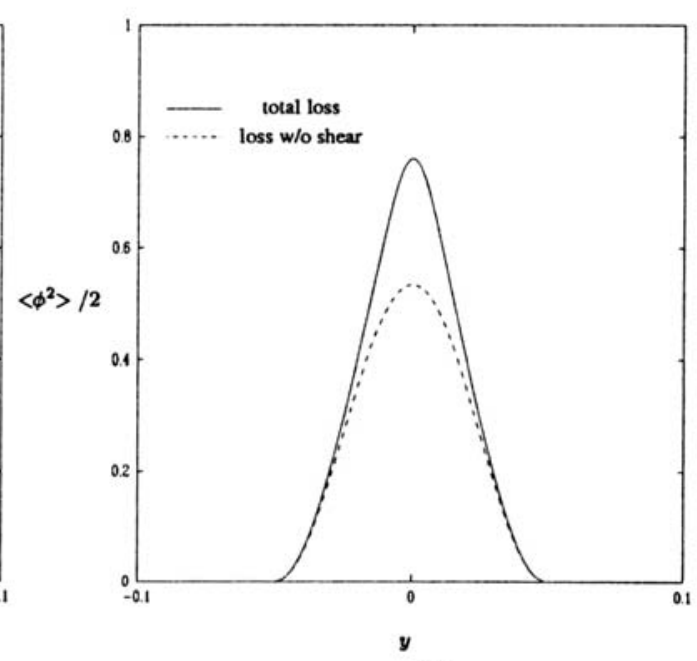

(b)

Fig. 8 Distribution of $\left\langle\phi_{1}^{2}>/ 2\right.$ for (a) a flow compensated gradient and (b) a bipolar gradient.

$$
\bar{u}=U \text { for } y>0 \text { and } \bar{u}=0 \text { for } \mathbf{y}<\mathbf{0} \text {. }
$$

Dashed lines represent the loss without the consideration of flow shear.

be different, its dimensionless form (normalized by $D^{2}$ ) remains the same. Therefore, it is possible to scale up or down the velocity if all the other variables are adjusted accordingly.

A constant eddy diffusivity can not describe the phase variation, and a positive eddy diffusivity can not explain the even echo rephasing. There are short periods of time after the first and second echoes that the phases is experiencing rephasing for small $\tau / T_{0}$. This rephasing disappears when $\tau / T_{0}>1$, that corresponds a truly random velocity during the application of magnetic gradients. The negative eddy diffusivity, which is an analogy of energy backscatter in turbulent theory, provides a mechanism to describe the even echo rephasing phenomenon. Clarification of eddy diffusivity is essential in identifying the quantities that were measured in experiments. It is clear from our study that the eddy diffusivity is not just a product of velocity fluctuation $\left\langle u^{\prime 2}\right\rangle$ and its characteristic time scale, but a more complex expression of $\left\langle u^{\prime 2}\right\rangle,\left\langle v^{\prime 2}\right\rangle$ and $\left\langle u^{\prime} v^{\prime}\right\rangle$. Especially in the near wall region and in the vicinity of a turbulent jet and a mixing layer, the high velocity gradient will amplify the effect of $\left\langle v^{\prime 2}\right\rangle$ and $\left\langle u^{\prime} v^{\prime}\right\rangle$. The major difference between the eddy diffusivity in the modified Bloch equation and in the heat/transfer in turbulent is that the latter is a scalar diffusion, while the former describes a duffusion of a vectorial quantity, the transverse mag- netization. Since the modulus of transverse magnetization for an individual particle is conserved, each component of transverse magnetization is not governed by a diffusion equation alone. As a result, a positive and constant eddy diffusivity is not able to explain the change of phase in turbulence unless the integral time scale is much smaller than $\tau$.

Table 1. Characteristic scales for turbulent pipe flow, [15-16]. $y=0$ denotes the center of channe.

\begin{tabular}{|l|l|l|l|l|l|c|l|l|l|}
\hline $\mathrm{y}$ & $L_{u}$ & $L_{v}$ & $\bar{u}$ & $u^{\prime}$ & $v^{\prime}$ & $-\left\langle u^{\prime} v^{\prime}\right\rangle$ & $\partial \bar{u} / \partial y$ & $T_{u}$ & $T_{v}$ \\
\hline 0.008 & 1.44 & 0.8 & 19.4 & 0.83 & 0.64 & 0.01 & 2 & 0.0774 & 0.0416 \\
\hline 0.247 & 1.76 & 0.8 & 17.8 & 1.3 & 0.76 & 0.47 & 12 & 0.096 & 0.0430 \\
\hline 0.451 & 3.2 & 0.96 & 12.0 & 2.7 & 0.42 & 0.6 & 120 & 0.264 & 0.08 \\
\hline 0487 & 3.2 & 0.96 & 6.2 & 2.1 & 0.14 & 0.2 & 320 & 0.520 & 0.152 \\
\hline
\end{tabular}

\section{REFERENCES}

1. J.-H. Gao, S. K. Holland, J. C. Gore, "Nuclear magnetic resonance signal from flow nuclei in rapid imaging using gradient echoes," Med. Phys. 15(6): 809-814 (1988).

2. H. Y. Carr, E. M. Purcell, "Effects of diffusion on free precession in nuclear magnetic resonance experiments," Phys. Rev. 94(3): 630-638 (1954).

3. J.-H. Gao, J. C. Gore, "Turbulent flow effects on NMR imaging: Measurement of turbulent intensity,"Med. Phys. 18(5): 1045-1051 (1991).

4. D. O. Kuethe, J.-H. Gao, "NMR signal loss from turbulence - model of time dependence compared 
with data," Phys. Rev. E 51(4): 3252-3262 (1995).

5. P. G. De Gennes, "Theory of spin echoes in a turbulent fluid,"Phys. Lett. A 29(1): 20-21 (1969).

6. K. Fukuda, A. Hirai, "A pulsed NMR study on the flow of fluids,"J. Phys. Soc. Jpn. 47(6): 1999-2006 (1979).

7. T.-Q. Li, J. D. Seymore, R. L. Powell, K. L. McCarthy, L. Odberg, M. J. McCarthy, "Turbulent pipe flow studied by time-averaged NMR imaging: measurements of velocity profile and turbulent intensity," Magn. Reson. Imag. 12(6): 923-934 (1994).

8. D. O. Kuethe, "Measuring distribution of diffusivity in turbulent fluids with magnetic resonance imaging," Phys. Rev. A 40(8): 4542-4551 (1989).

9. A. J. Favre, "Review on space-time correlations in turbulent fluids," J. Appl. Mech. 241-257 (1965).

10. W. D. McComb, The Physics of Fluid Turbulence, (Oxford Univ. Press, New York, 1990), pp. 436459.

11. R. P. Spielmann, R. P., O. Schneider, F. Thiele, M. Heller, E. Bucheler, "Appearance of poststenotic jets in MRI: dependence of flow velocity and on imaging parameters," Magn. Reson. Imag. 9: 67-72 (1991).

12. A. J. Evans, R. A. Blinder, R. J. Herfkens, C. E. Spritzer, D. O. Kuethe, E. K. Fram, L. W. Hedlund, "Effects of turbulence on signal intensity in gradient echo images,"Invest. Radiol. 23(7): 512-518 (1988).

13. S. N. Urchuk, D. B. Plewes, "Mechanisms of flowinduced signal loss in MR angiography," J. Magn. Reson. Imag. 2: 453-462 (1992).

14. J. N. Oshinski, D. N. Ku, R. I. Pettigrew, "Turbulent fluctuation velocity - the most significant determinant of signal loss in stenotic vessels," Magn. Reson. Med. 33: 193-199 (1995).

15. P. K. Yeung, S. B. Pope, "An algorithm for tracking fluid particles in numerical simulations of homogeneous turbulence," J. Comput. Phys. 79: 373 (1988).

16. J. G. M. Eggels, F. Unger, M. H. Weiss, J. Westerweel, R. J. Adrian, R. Friedrich, F. T. M. Nieuwstadt, "Fully developed turbulent pipe flow: a comparison between direct numerical simulation and experiment," J. Fluid. Mech. 268: 175-209 (1994).

17. J. Westerweel, A. A. Draad, J. G. Th. van der Hoeven, J. van Oord,"Measurement of fullydeveloped turbulent pipe flow with digital particle image velocimetry," Exp. Fluids 20: 165-177 (1996).

18. J. C. H. Fung, "Shear flow turbulence structure and its Lagrangian statistics,"Fluid Dynamics Res. 17: 147-180 (1996).

19. P. S. Bernard, R. A. Handler, "Reynolds stress and the physics of turbulent momentum transport," J.
Fluid Mech. 220: 99-124 (1990).

20. P. A. Chevrin, P. A., H. L. Petrie, S. Deutsch, "The structure of Reynolds stress in the near-wall region of a fully developed turbulent pipe flow," Exp. Fluid, 13(6): 405-413 (1992).

21. M. Van Dyke, An Album of Fluid Motion, (Parabolic Press, Stanford, California, 1982), pp. 60-70.

22. R. P. Spielmann, J. Zhen, H. J. Triebel, V. Nicolas, M. Heller,E. Bücheler, "Magnetic resonance imaging and pulsed Doppler sonography of poststenotic jets: correlation between signal void and flow velocity distribution," Magn. Reson. Imag. 10: 893901 (1992).

23. R. D. Gould, W. H. Stevenson, H. D. Thompson, "Investigation of turbulent Transport in an axisymmetric sudden expansion," AIAA J. 28(2): 276283 (1990).

24. J. C. Gatenby, J. C. Gore, "Characterization of turbulent flows by NMR measurements with pulsed gradients," J. Magn. Reson. A 1 10: 26-32 (1994).

25. J. C. Gatenby, J. C. Gore, "Echo-planar-imaging studies of turbulent flow"J. Magn. Reson. A. 121: 193-200 (1996).

\section{Apppendix 1. A bipolar gradient (BP)}

If $R_{n v}^{\prime}=e^{-|t| ! T_{v}}$ and $R_{m, t}^{\prime}=e^{-|t| / T_{u}}$

$$
\begin{gathered}
f_{v}^{h p}\left(w=\tau / T_{v}\right)=2\left(w^{2}-1\right) e^{-2 w}+\left(-6+4 w+2 w^{2}\right. \\
\left.-\frac{4}{3} w^{3}-w^{4}+\frac{23}{15} w^{5}\right)+\left(8-4 w^{2}\right) e^{-w},
\end{gathered}
$$

and

$$
f_{u}^{b p}\left(w=\tau / T_{u}\right)=\frac{4}{3} w^{3}-4 w+\left(6-8 e^{-w}+2 e^{-2 w}\right) .
$$

When $\tau<<T_{u,}, T_{v}$ and $T_{n v}$, then

$$
<\left.\not\right|^{2}>/\left(\mathcal{L} G_{x}\right)^{2}=\tau^{4}<u^{2}>+\left(\frac{d \bar{u}}{d y}\right)^{2} \tau^{6}<v^{\prime 2}>-2<u v^{\prime}>\tau^{s} \frac{c \bar{u}}{d y} \text {. }
$$

Note that the first term corresponds to the result in Gao $\&$ Core [3]:

$\frac{<\phi_{1}^{2}>}{2}=\frac{1}{2}\left(\gamma G_{x}\right)^{2} \tau^{4}<u^{\prime 2}>$.

Appendix 2. A simple pulse gradient (SP)

For a simple pulse gradient,

$$
\begin{aligned}
& f_{u}^{s}\left(\tau, T_{m m}\right)=\frac{1}{T_{u}^{4}} \oint^{\tau}\left\lceil R_{m u}^{\prime \prime{ }^{\prime}} t t^{\prime} d t d t^{\prime},\right. \\
& f_{v}^{s p}\left(\tau, T_{v}\right)=\frac{1}{4 T_{v}^{6}} \int_{b}^{c} \int_{b u}^{\prime-t^{\prime}} t^{2} t^{\prime 2} d t d t^{\prime}, \\
& f_{m,}^{s p}\left(\tau, T_{w}\right)=\frac{1}{T_{w}^{5}} \oint_{b}^{\tau}\left\lceil<u_{,}^{\prime} v_{i^{\prime}}^{\prime}>t^{\prime 2} t d t d t^{\prime}\right.
\end{aligned}
$$


(22)

If $R_{u u}^{\prime}=e^{-\mid t ! / T_{u}}$ and $R_{v v}^{\prime}=e^{-\left|r_{i}\right| T_{v}}$, then

$f_{u}^{s p}\left(w=\frac{\tau}{T_{u}}\right)=-2(w+1) e^{-w}+\left(\frac{2}{3} w^{3}-w^{2}+2\right)$,

and

$$
\begin{aligned}
f_{v}^{s p}\left(w=\frac{\tau}{T_{v}}\right)= & \frac{1}{2}\left(w^{2}+w+1\right) e^{w} \\
& +\left(\frac{1}{20} w^{5}-\frac{1}{8} w^{4}+\frac{1}{6} w^{3}-1\right) .
\end{aligned}
$$

Furthermore, if

$$
\tau \ll T_{u}, T_{y} \text { and } T_{w} \text {, then } f_{u}^{s p}(w) \approx w^{4} / 4
$$

and $f_{v}^{s p}(w) \approx w^{6} / 36$.

\section{Appendix 3. A velocity-compendsated pulse (FC)}

For a velocity compensated gradient, Eqn. (4) becomes

$$
\frac{1}{\gamma G_{x}} \frac{d \phi_{0}}{d t}= \begin{cases}\bar{u}_{t} t & \text { if } 0<t<\tau, \\ \bar{u}_{t}(2 \tau-t) & \text { if } \tau<t<3 \tau \\ \bar{u}_{t}(t-4 \tau) & \text { if } 3 \tau<t<4 \tau\end{cases}
$$

The integration of the equation above yields

$$
\phi_{0}= \begin{cases}\gamma G_{x} \bar{u}_{t} \frac{t^{2}}{2} & \text { if } 0<t<\tau, \\ -\gamma G_{x} \bar{u}_{t}\left(\frac{(2 \tau-t)^{2}}{2}-\tau^{2}\right) & \text { if } \tau<t<3 \tau, \\ \gamma G_{x} \bar{u}_{t} \frac{(t-4 \tau)^{2}}{2} & \text { if } 3 \tau<t<4 \tau .\end{cases}
$$

Substituting $\phi_{0}$ into Eqn. (5), we have

$$
\frac{1}{\mathcal{G}_{x}} \frac{d \phi_{1}}{d t}= \begin{cases}u_{t}^{\prime} t-v_{\prime}^{\prime} \frac{d \bar{u}}{d y} \frac{t^{2}}{2} & \text { if } 0<t<\tau, \\ u_{t}^{\prime}(2 \tau-t)+v_{t}^{\prime} \frac{d \bar{u}}{d y}\left(\frac{(2 \tau-t)^{2}}{2}-\tau^{2}\right) & \text { if } \tau<t<3 \tau, \\ u_{t}^{\prime}(t-4 \tau)-v_{t}^{\prime} \frac{d \bar{u}}{d y} \frac{(t-4 \tau)^{2}}{2} & \text { if } 3 \tau<t<4 \tau .\end{cases}
$$

$\phi_{1}(t=4 \tau)$ should be

$$
\begin{aligned}
\phi_{1}(t & =4 \tau)=-\frac{\chi G_{x}}{2} \frac{d \bar{u}}{d y} \int_{b}\left\{\left[v_{t}^{\prime}+v_{4 \tau-1}^{\prime}\right] t^{2}-\left[v_{2 \tau-t}^{\prime}+v_{t+2 \tau}^{\prime}\right]\left(t^{2}-2 \tau^{2}\right)\right\} d t \\
& +\gamma G_{x} \int_{b}^{r}\left[\left(u_{,}^{\prime}-u_{4 \tau,}^{\prime}\right)+\left(u_{2 r-1}^{\prime}-u_{2 r+1}^{\prime}\right)\right] t d t
\end{aligned}
$$

The ensemble average of $\phi_{1}$ is

$$
\begin{aligned}
& <\phi_{1}^{2}>/\left(\gamma G_{x}\right)^{2} \\
& =\frac{1}{2}\left(\frac{d \bar{u}}{d y}\right)^{2}<v^{\prime 2}>\iint\left[t^{2} t^{\prime 2}\left(R_{v v}^{t-t}+R_{v v}^{d r-t-t^{\prime}}\right)\right. \\
& \quad+\left(t^{2}-2 \tau^{2}\right)\left(t^{\prime 2}-2 \tau^{2}\right)\left(R_{v+}^{\prime-t^{\prime}}+R_{v v}^{i+t}\right)
\end{aligned}
$$

$$
\begin{aligned}
& \left.-2\left(t^{2}-2 \tau^{2}\right) t^{\prime 2}\left(R_{w}^{2 r-t-t^{\prime}}+R_{w}^{2 r-t+t}\right)\right] d t d t^{\prime} \\
& +2<u^{\prime 2}>\int_{b}^{T}\left[2 R_{u u}^{t-t^{\prime}}+2 R_{u t}^{2 r-t-t^{\prime}}-R_{u u}^{2 \tau+t-t^{\prime}}-R_{u u}^{2 \tau-t+t^{\prime}}\right. \\
& \left.\left.-R_{u w}^{t r-t-t^{\prime}}-R_{u v}^{i+t^{\prime}}\right] t t^{\prime} d t d t^{\prime}-\left(\frac{d \bar{u}}{d y}\right)<u^{\prime} v^{\prime}\right\rangle
\end{aligned}
$$

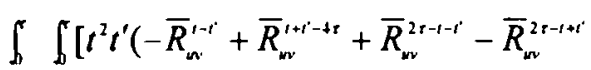

$$
\begin{aligned}
& \left.-\left(t^{2}-2 \tau^{2}\right) t^{\prime}\left(\bar{R}_{\mathrm{vv}}^{t-\tau}-\bar{R}_{\mathrm{uv}}^{t+t}-\bar{R}_{\mathrm{uv}}^{2 \tau-t-t^{\prime}}-\bar{R}_{\mathrm{uv}}^{2 \tau-t+t}\right)\right] d t d t^{\prime} \\
& =T_{v}^{6}\left(\frac{d \bar{u}}{d y}\right)^{2}<v^{\prime 2}>f_{v}^{f c}\left(\tau, T_{v}+<u^{\prime 2}>T_{u}^{4} f_{u}^{f k}\left(\tau, T_{u}\right)\right. \\
& -\left(\frac{d \bar{u}}{d y}\right)<u^{\prime} v^{\prime}>T_{w v}^{s} f_{w v}^{s c}\left(\tau, T_{w}\right)
\end{aligned}
$$

where $\bar{R}_{w v}^{\prime}=R_{w v}^{\prime}-R_{w v}^{-t}$ and superscript $f c$ denotes flowcompensation. Note that if $R_{u v}$ is an even function, then $\bar{R}_{\alpha}=0$.

Using $R_{v v}^{\prime}=e^{-1 k / r}, f_{v}^{\prime c}\left(\tau, T_{v}\right)$ becomes

$f_{v}^{f c}\left(\tau, T_{v}\right)=f_{v}^{\prime c}\left(\tau / T_{v}\right)=f_{v}^{\prime c}(w)$

$=\frac{46}{15} w^{5}+w^{4}\left(2 e^{-3 w}-2 e^{-x}\right)$

$+w^{3}\left(-\frac{8}{3}+4 e^{-3 w}-8 e^{-2 w}+4 e^{-w}\right)+8 w$

$+\left(-10+2 e^{-4 w}-8 e^{-3 w}+8 e^{-2 w}+8 e^{-\omega}\right)$;

similarly, $f_{u}^{f c}\left(\tau, T_{n}\right)$ yields

$f_{u}^{\prime c}\left(\tau, T_{u}\right)=f_{*}^{\prime c}\left(\tau / T_{u}\right)=f_{*}^{\prime c}(w)$

$=\frac{8}{3} w^{3}-8 w+\left(10-2 e^{-4 w}+8 e^{-3 w}-8 e^{-2 w}-8 e^{-w}\right.$

if $R_{w u}^{i}=e^{-\tau, t T}$

For $w<1, f_{v}^{f c} \approx 4 w^{6}$ and $f_{v}^{c c} \approx 46 w^{s} / 15$. Note that both $f_{u}^{s p}$ and $f_{u}^{\text {hp }}$ approach $w^{4}$ asymptotically, but $f_{u}^{f t} \approx w^{s}$ for $w<<1$. Thus, UFT ${ }^{f c} \approx<u^{\prime 2}>\tau^{5} / T_{u}$. 